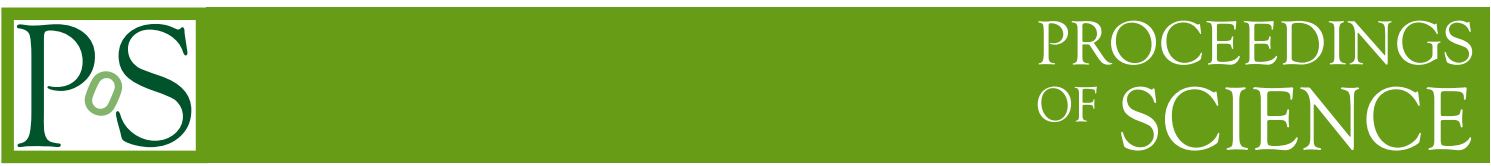

\title{
A silicon sensor study for the ILD ECAL
}

Tatsuhiko Tomita*

Department of Physics, Kyushu University

E-mail: Eomitadepp.phys.kyushu-u.ac.jp

Hiroto Hirai, Kiyotomo Kawagoe, Yohei Miyazaki, Yuji Sudo, Taikan Suehara, Hiroki Sumida, Shusuke Takada, Hiraku Ueno, Tamaki Yoshioka.

Department of Physics, Kyushu University

The International Large Detector (ILD) is a proposed detector for the International Linear Collider (ILC). It has been designed to achieve an excellent jet energy resolution by using Particle Flow Algorithm (PFA), which relies on the ability to separate nearby particles within a jet. PFA requires the calorimeters with high granularity. A sampling calorimeter with tungsten plates and silicon sensors is proposed for the Electromagnetic Calorimeter (ECAL). Thirty layers of tungsten plates, with total thickness of about $24 \mathrm{X}_{0}$, are chosen for small Moliere radius to minimize overlap of electromagnetic showers. Fine granularity is achieved by using silicon sensors having 256 pixels of $5.5 \times 5.5 \mathrm{~mm}^{2}$ in an area of $9 \times 9 \mathrm{~cm}^{2}$. The total number of readout channels amounts to the order of $10^{8}$. We have measured various properties of these prototype sensors: the leakage current, capacitance, and full depletion voltage. To optimize the sensor design, we have also examined relation of the guard ring structure and the cross talk between pixels using an infrared laser system.

Technology and Instrumentation in Particle Physics 2014,

2-6 June, 2014

Amsterdam, the Netherlands

* Speaker. 


\section{Introduction}

The International Linear Collider (ILC) [U, [ [] is a future high energy $\mathrm{e}^{+} \mathrm{e}^{-}$collider to explore new physics beyond the standard model through precision measurements of the Higgs boson and the top quark as well as study of other electroweak processes. Direct discovery of new particles can also be expected within its center of mass energy. As most of important physics processes include multi-jet final states, an ILD detector should have unprecedentedly good jet energy resolution (3$4 \%$ for a $100 \mathrm{GeV}$ jet). Particle Flow Algorithm (PFA) [3] has been proposed and developed to achieve such good resolution, where separation of individual incident particles in the calorimeters is essential. To this purpose, an electromagnetic calorimeter (ECAL) is required to have a granularity of order $5 \times 5 \mathrm{~mm}^{2}$. In the International Large Detector (ILD) [四] concept, a silicon-tungsten sampling ECAL is proposed. Tungsten absorber plates are used to have small Moliere radius to minimize overlaps of electromagnetic showers. Silicon sensors having small pixels are used to realize the fine granularity required for PFA. In this report, some results on the R\&D study of the silicon sensors are presented.

\section{Silicon sensors}

We have designed and produced prototype silicon sensors for the ILD ECAL and in the CALICE collaboration [5], manufactured by Hamamatsu Photonics (HPK). A silicon sensor is a silicon PIN diode with thin $\mathrm{P}^{+}$pixels on $\mathrm{N}^{+}$substrate. The thickness of the sensors is $320 \mu \mathrm{m}$ and the size is $9 \times 9 \mathrm{~cm}^{2}$ segmented into $16 \times 16$ pixels of size $5.5 \times 5.5 \mathrm{~mm}^{2}$ (Figure 1). In addition to the normal-size $(16 \times 16$ pixels) sensors, small-size test sensors with $4 \times 4$ or $3 \times 3$ pixels are specially produced. There are several variations of small-size sensors: 4 types of guard ring structure (Figure 2) and 2 kinds of doping densities.

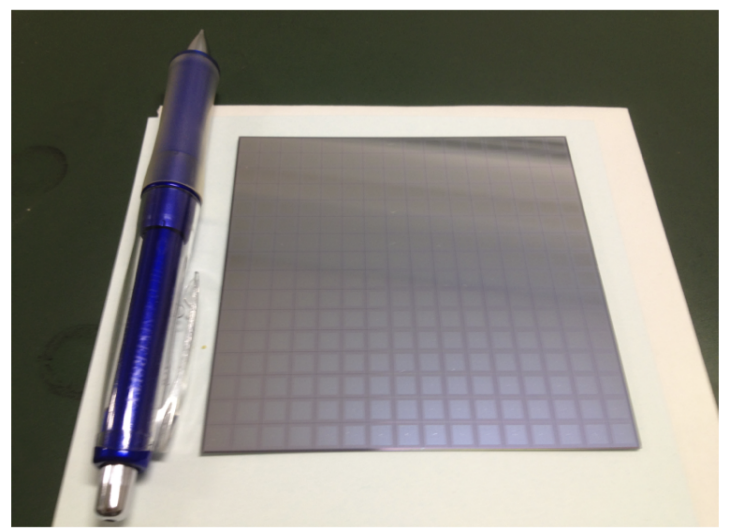

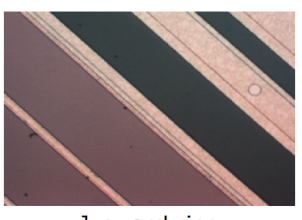

1 guard ring

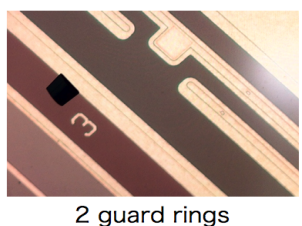

2 guard rings

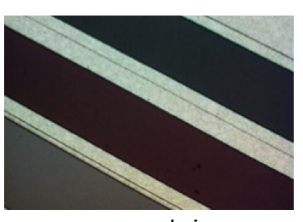

no guard ring

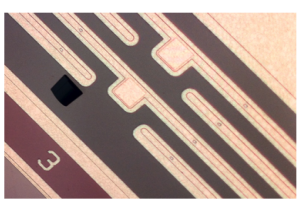

4 guard rings
Figure 2: Guard ring structures: the guard ring width is $80 \mu \mathrm{m}$ (1 guard ring), or $20 \mu \mathrm{m}$ (2, 4 guard rings).

Figure 1: A silicon sensor having $16 \times 16$ pixels. 1 guard ring structure has one continuous guard ring along circumference of chip, but 2 and 4 guard ring structure have split guard rings. 
The guard rings can adjust the electric field nearby the edge of the sensors, so that the leakage current is decreased. The guard rings, however, make non-active area around the edge, which should be minimized. In order to optimize the guard ring structure, various guard ring types, including split guard rings and no guard rings, are studied. We measured and compared their basic properties of the test sensors, such as leakage current, capacitance and sensor response to injected charge, to optimize the sensor design for the ILD ECAL.

\section{Basic properties: leakage current and capacitance}

We measured the capacitance and the leakage current of the sensors in a condition of $20{ }^{\circ} \mathrm{C}$ and $50 \%$ humidity. A copper plate is used to combine signals from all pixels in a sensor to one readout channel. The data taking were automated and controlled by LabVIEW.
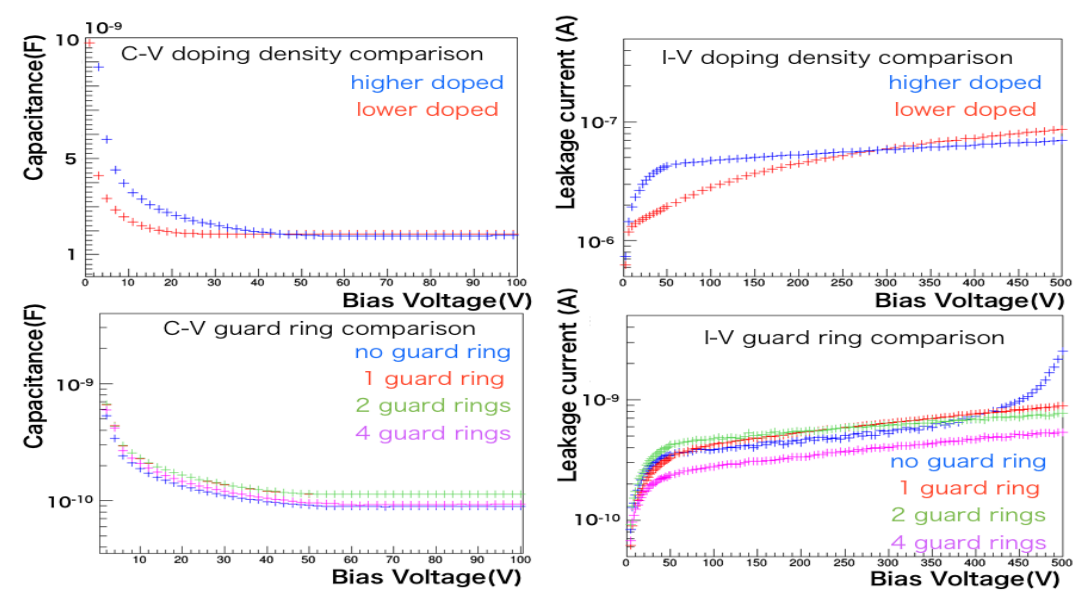

Figure 3: Results of basic measurements of silicon sensors: left two plots show the results of capacitance measurement, and right two plots show the results of leakage current of sensors. Upper two plots are comparison between different doping densities, bottom two plots are between different guard ring structures. The results of both measurements were divided by its number of pixels.

Results of the capacitance and the leakage current measurements are shown in Figure 3. The capacitance measurements show that the guard ring structure does not affect the capacitance of sensors but the doping density can change the full depletion voltage. Sensors with lower doping density are preferred because of the lower full depletion voltage, that leads to reduction of power consumption and heat emission of sensor operation. Results of the leakage current show that the sensors with lower doping density have less leakage current up to the bias voltage of $300 \mathrm{~V}$, which is much higher than the full depletion voltage of the sensors. As expected, sensors with split guard rings ( 2,4 guard rings) show lower leakage current than that of 1 continuous guard ring type, but the difference is not significant.

\section{Response to an infrared laser: study of guard ring effect}

A known problem of silicon-pad sensors is cross talks between pixels mediated by guard rings. The effects are investigated by using a YAG infrared laser system. The wave length of the laser 
light is $1064 \mathrm{~nm}$ corresponding to a photon energy of $1.16 \mathrm{eV}$. The photon energy is greater than the silicon band gap energy of $1.12 \mathrm{eV}$, enough to create an electron-hole pair, and the absorption length of the sensor to the photon is long enough. An optical lens focuses the laser light on the sensor surface with a spot size less than $20 \mu \mathrm{m}$. The laser light is shot between a pixel and the guard ring to investigate the cross talk effect related to the guard ring structure.

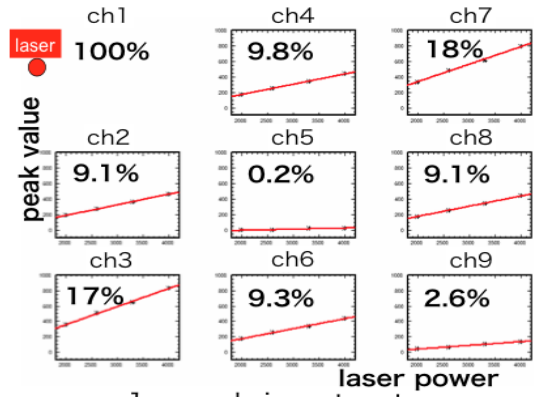

1 guard ring structure

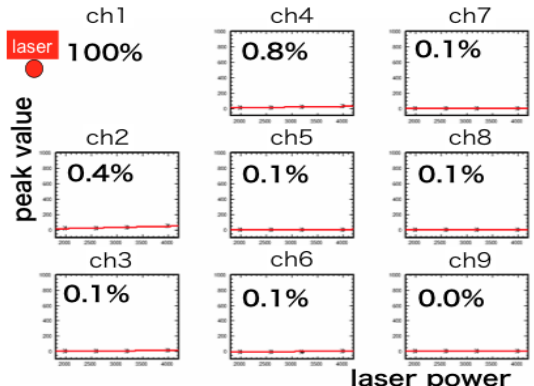

4 guard rings structure

Figure 4: Left top pixels are reference channels, and the percentage of each channel is ratio of cross talk. Left plot shows the result of 1 guard ring structure, and right plot shows 4 guard ring structure's result.

Figure 4 shows the response of each pixel, and the cross talk signals are clearly observed for the sensor with 1 guard ring structure, because the guard ring surrounds the pixels along circumference of sensor. Since 2 and 4 guard ring structures have split guard rings, they have little cross talk along the guard rings. No guard ring structure showed no cross talk as expected. We should use no, 2 or 4 guard ring structure to reduce unnecessary cross talk mediated by the guard rings.

\section{Conclusions and prospects}

We measured basic properties of the prototype silicon sensors and response to a focused infrared laser light. As a result of these measurements, we may conclude that the sensor with lower doping density is preferred for low voltage operation, and no guard ring structure is preferred to minimize the non-sensitive area and to reduce cross talk effect along with the guard ring. We need, however, further studies to finalized the sensor design: radiation hardness, cross talk between pixels, and so on.

\section{References}

[1] T,Behnke, James E. Brau, B. Foster, J. Fuster, M. Harrison, et al, The International Linear Collider Technical Design Report - Volume 1: Executive Summary. 2013.

[2] T,Behnke, James E. Brau, Philip N. Burrows, J. Fuster, M. Peskin, et al. The International Linear Collider Technical Design Report - Volume 4: Detectors. 2013.

[3] M.A. Thomson. Particle Flow Calorimetry and the PandoraPFA Algorithm. Nucl. Instrum. and Meth. A611 (2009) 25-40.

[4] ILD concept : http://ilcild.org/

[5] CALICE wiki : https://twiki.cern.ch/twiki/bin/view/CALICE/WebHome 